\title{
Spectrum resource assignment method considering adjacent link conditions for AR requests in $\mathrm{EON}$
}

\author{
Yoichiro Arii $^{1 a)}$, Ken-ichi Baba ${ }^{1)}$ \\ ${ }^{1}$ Department of Information and Communications Engineering, Kogakuin- \\ University \\ 1-24-2 Nishi-shinjuku,Shinjuku,Tokyo 163-8677 \\ a)cm21002@ns.kogakuin.ac.jp
}

Abstract: Elastic optical networks (EONs) have attracted attention as a means of accommodating the rapidly increasing traffic in core networks. In EONs, the RMSA algorithm is implemented to flexibly allocate resources to optical paths. However, the application of EONs is limited because of the fragmentation of spectrum resources, which hinders further allocation of resources to new optical paths. Subsequently, the Min-RDDR method has been proposed to allocate resources for advance reservation requests. It defined an evaluation formula for calculating a metric to suppress fragmentation. Herein, we modified the Min-RDDR method and proposed a spectrum allocation method to further suppress fragmentation, wherein resources were considered not only within links but also between adjacent links. The simulation results confirmed that the proposed method causes a $47 \%$ reduction of request blocking probability, verifying its effectiveness toward fragmentation suppression.

Keywords: Elastic Optical Network, Advance Reservation, RMSA, Spectrum Assignment

Classification: Photonic Network Control

\section{References}

[1] B. Chatterjee, S. Ba, and E. Oki, "Fragmentation problems and management approaches in elastic optical networks: A survey," IEEE Communications Surveys Tutorials, vol. 20, no. 99, pp. 183-210, Feb. 2018.

[2] Y. Wang, "A research on spectrum defragmentation algorithms in elastic optical network," Proc. of World Symposium on Communication Engineering (WSCE2019), pp. 78-81, Nov. 2019.

[3] Y. Yin, M. Zhang, Z. Zhu, and S. J. B. Yoo, "Fragmentation-aware routing, modulation and spectrum assignment algorithms in elastic optical networks," Proc. of IEEE Optical Fiber Communication, pp. 1-3, Mar. 2013.

[4] H. Chen, Y. Zhao, J. Zhang, R. He, W. Wang, J. Wu, Y. Wang, Y. Ji, H. Zheng, Y. Li, and B. Hou, "Time-spectrum consecutiveness based scheduling with advance reservation in elastic optical networks," IEEE Communications Letter, vol. 19, no. 1, pp. 70-73, Jan. 2015. 
[5] S. Sugihara, Y. Hirota, S. Fujii, H. Tode, and T. Watanabe, "Routingand spectrum allocation method for immediate reservation and advance reservation requests in elastic optical networks," Proc. of Photonic Switching(PS), pp. 178-180, Sep. 2015.

[6] X. Li, J. Yuan, Q. Zhang, Z. Ren, and L. Yang, "Farsighted spectrum resource assignment method for advance reservation requests in elastic optical networks," IEEE Access, vol. 7, pp. 167836-167846, Nov. 2019.

[7] J. Yen, "Finding the k shortest loopless paths in a network," Manage.Sci., vol. 17, pp. 712-716, July 1971.

\section{Introduction}

With the rapid development of cloud/edge computing, 5G communications, and other technologies, traffic has been rapidly growing in the backbone network. As a solution, elastic optical networks (EONs) have attracted attention because they can efficiently use spectrum resources to accommodate the increasing traffic. Furthermore, flexible allocation is possible for different bandwidths depending on the type of application. Based on the kind of application, the corresponding connection requests may either be immediate reservation (IR) or advance reservation (AR) ones. For example, video conferencing applications are treated as IR traffic, and data backups between data centers as AR traffic.

The fragmentation of spectrum resources occurs between used slots and empty slots, during the allocation of frequency slots to optical paths. To address this problem, Ref. (1) proposed the Min-RDDR method as a means of allocation for AR requests, which reserved resources in advance in the EON. This method allocates spectrum resources efficiently by using the cost calculated from the reservation conditions in a candidate path.

Herein, we proposed a spectrum allocation method that could increase the number of AR requests to which resources can be allocated, by saving as much common free spectrum resources as possible, for future requests on adjacent links. We modified the conventional Min-RDDR method by introducing a cost function that considers adjacent link conditions.

\section{Related works}

EONs use spectrum resources more flexibly and efficiently, but optical paths need to satisfy spectrum continuity and spectrum contiguity. Spectrum fragmentation prevents a new optical path from being accommodated $[1,2]$. As a solution to this problem, many RMSA algorithms have been proposed to reduce spectrum fragmentation and use resources efficiently. In [3], they proposed a spectrum allocation method that checks the condition of spectrum resources and considers the degree of fragmentation within a link and adjacent links. In [4], they proposed an RSA method based on a scheduling algorithm that reduces fragmentation in both the spectrum and time dimension for AR requests in WDM networks. In [5], they proposed an RMSA algorithm that 
sets up a priority area, which is divided depending on the required bandwidth. Many of the proposed algorithms for AR requests introduce metrics to measure the condition of spectrum resources in a link. However, many of them consider only a part of the situation of the spectrum resources; the optimization of the resource utilization method is limited. Therefore, in [6], they proposed an allocation method called the Min-RDDR algorithm that introduces a new metric, which reflects the condition of the entire of the spectrum resources in a link.

\section{Proposed method}

In the Min-RDDR method, the assigned frequency block is determined using an evaluation formula for calculating metric $M$; the formula sums up four factors calculated from the condition in the links of the candidate routes. Figure 1(a) shows an example of calculating each factor. Firstly, $R_{c}$ is calculated by multiplying the required number of frequency slots and time slots, by the number of hops in the candidate route. $D_{f}$ indicates the distance between both ends of the frequency slot, and $D_{t}$ indicates the difference between the communication start time that can be started and the communication start time in the allocated slot. Finally, $R_{d}$ indicates the number of empty slots around allocated block.

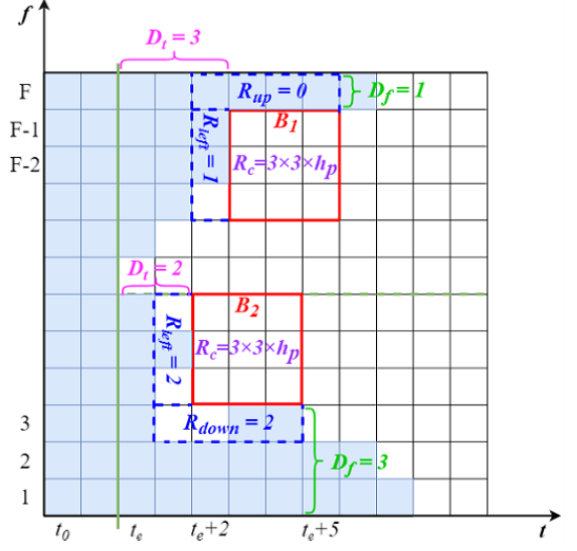

(a) Factor calculation example in MinRDDR

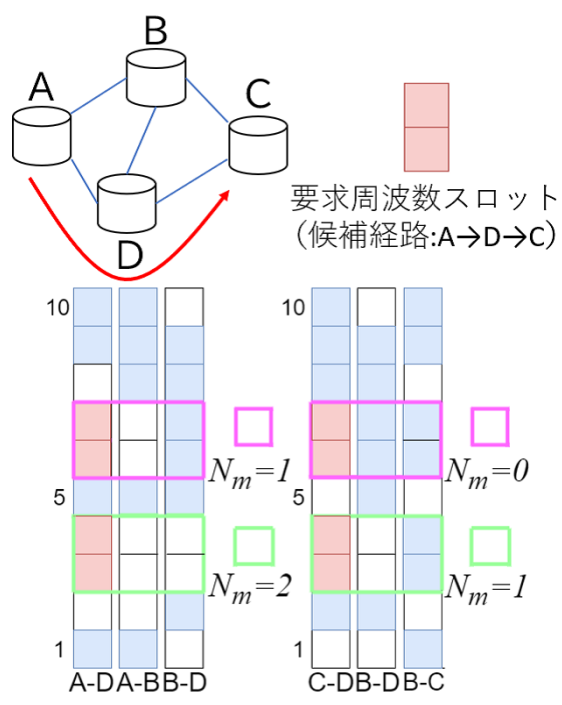

(b) $N_{m}$ calculation example

Fig. 1: Factor calculation example

In our proposed method, we improved the metric calculation formula of the Min-RDDR method. We introduced a new factor, $N_{m}$, which considers the condition of adjacent links. An adjacent link refers to all links passing through a single node connecting the link. Examining the condition of adjacent links enables the consequent scrutiny of the fragmentation between them. Therefore, the current method increases the number of AR requests to which resources can be allocated. The evaluation formula in the proposed 
method is given in Eq. (1), wherein, $a, b, c, d$, and $e$ represent the coefficients for the weighting factor.

$$
M=a R_{c}+b D_{f}+c D_{t}+d R_{d}+e N_{m}
$$

$N_{m}$ is a new factor in the proposed method. $N_{m}$ is calculated by the following equation.

$$
N_{m}=\frac{\sum_{l=1}^{n} M_{l}}{n}
$$

In Eq. (2), $n$ indicates the number of adjacent links and $M_{l}$ indicates the number of empty slots in the allocated slots of the adjacent link 1.

Figure 1(b) shows an example of calculating $N_{m}$; for the sake of simplicity, we have not considered time slots here. As shown in the figure, the frequency slots in link AD and the link CD are assigned. The adjacent links of link AD are indicated as links $\mathrm{AB}$ and $\mathrm{BD}$, and those of link $\mathrm{CD}$ as links $\mathrm{BC}$ and $\mathrm{BD}$. Furthermore, the free frequency blocks that can be assigned are slot numbers 3, 4 (green block) and 6, 7 (pink block); hence, we compare these blocks. If assigned to the green block, link $\mathrm{AD}$ has two adjacent links with a total of four empty slots, and link CD has two adjacent links with a total of two empty slots; therefore, total $N_{m}$ in the green block is equal to 3. Similarly, if assigned to the pink block, link $\mathrm{AD}$ has two adjacent links with a total of two empty slots, and link CD has two adjacent links without any empty slots; therefore, total $N_{m}$ in the pink block is equal to 1 . Because $N_{m}$ in the pink block is less than that in the green block, the pink block was chosen for assignment.

\section{Performance evaluation}

\subsection{Simulation settings}

We evaluated the performance of our proposed method using simulations. We used NSFNET with 21 links and 14 nodes as the network topology. Each frequency slot was set to $12.5 \mathrm{GHz}$, and the number of slots in each link was set to 320 . Additionally, the spectrum resources were divided with respect to time, and each time slot was set to $10 \mathrm{~min}$. The candidate route connecting the source node and the destination node used the Yen's algorithm [7], which searches up to the $k$-th shortest route, and $k$ was set to 3 . The modulation methods used were BPSK, QPSK, 8-QAM, and 16-QAM.

In this simulation, the request arrival rate follows a Poisson distribution, and the duration follows an exponential distribution with an average of 10 time slots. 100,000 requests were generated under the given load. The required capacity for each request varied from 25 to $250 \mathrm{Gbps}$, the communication start time varied from 1 to 30 , and the window size of the communication start time varied from 1 to 50, with uniform random numbers.

\subsection{Normalization process}

The evaluation formula for normalization is shown in the Eq. 3

$$
M=a\left(f R_{c}\right)+b\left(g D_{f}\right)+c\left(h D_{t}\right)+d\left(i R_{d}\right)+e\left(j N_{m}\right)
$$


By simulation, the average value when the frequency block was determined were $R_{c}=168, D_{f}=54, D_{t}=16, R_{d}=8 a n d N_{m}=50$, respectively. From this result, in order to do the average value of each factor to 100 , we set $f=0.60, g=1.85, h=6.66, i=12.50, j=2$.

\subsection{Comparison with benchmarks}

Figure 2(a) shows the request blocking probability of the entire network for each arrival rate. We compared the conventional, proposed, and normalized methods. As shown in the figure, the proposed method reduced the request blocking probability when compared with the conventional method. For example, when the offered load was 230 erlangs, the request blocking probability was reduced from 0.002524 to 0.00147 , which led to $47 \%$ reduction. This improvement was attributed to the reduction of the fragmentation of spectrum resources between adjacent links. The normalized method decreased the request blocking probability to a larger degree than the proposed method. For example, when the offered load was 230 erlangs, the request blocking probability was reduced from 0.002524 to 0.00066 , which resulted in $74 \%$ reduction. This is because in the evaluation formulas of the conventional and proposed methods, the influence is different for each factor. Therefore,

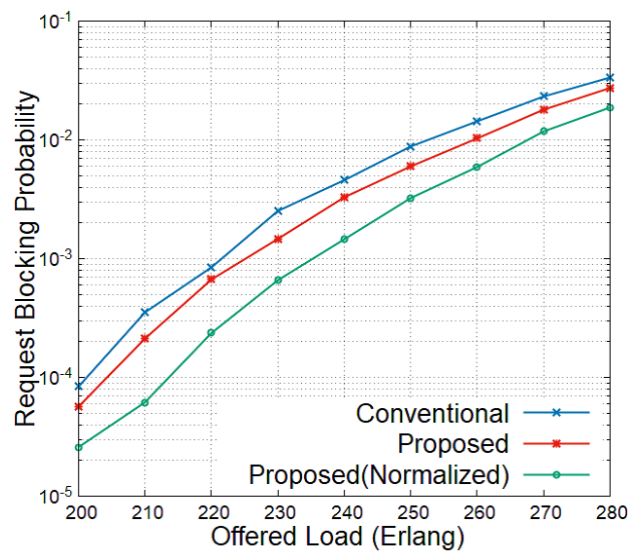

(a) Request blocking probability

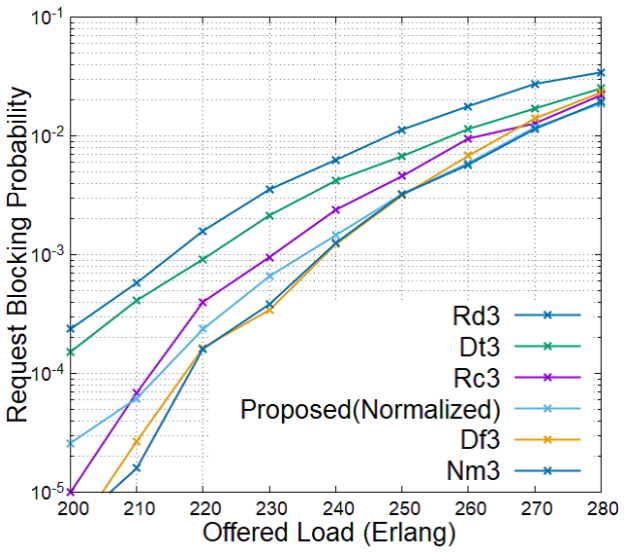

(b) Request blocking probability when each factor is tripled

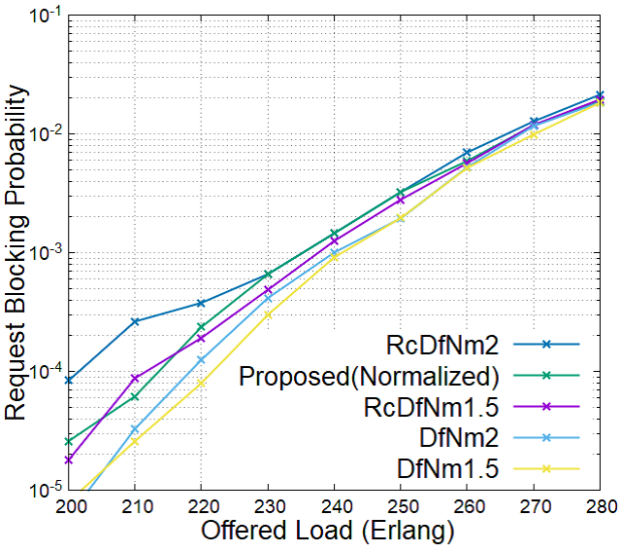

(c) Request blocking probability when combining factors

Fig. 2: In the NSFNET 
it is considered that the determination of the frequency block depends on one factor, or that one factor does not affect the allocation at all. By performing the normalization process, the weights of all the factors were made uniform and each factor was taken into consideration. Therefore, the normalized method enabled efficient allocation and reduced the request blocking probability.

The coefficients were examined using the normalized evaluation formula. For each factor, we investigated the request blocking probability when the influence was increased. Figure 2(b) shows the request blocking probability when the value of each factor was tripled. As seen in the figure, the request blocking probability decreases in the following order when only the Rd value is tripled in the evaluation formula: $R_{d}, D_{t}, R_{c}, D_{f}$, and $N_{m}$. Particularly, the high request blocking probabilities of $D_{t}$ and $R_{d}$ were remarkable; thus, we decided to exclude them and combine the remaining three factors to strengthen their influence.

Figure 2(c) shows the request blocking probability when $R_{c}, D_{f}$, and $N_{m}$ are 1.5 and 2 times their original values and when $D_{t}$ and $R_{d}$ are 1.5 and 2 times their original values. For example, $R_{c} D_{f} N_{m} 2$ refers to 2 -times higher $R_{c}, D_{f}$, and $N_{m}$. As seen in the figure, the influence of $D_{f}$ and $N_{m}$ is stronger than that of $R_{c}, D_{f}$, and $N_{m}$; the request blocking probability at that time is also reduced. Also, comparing the case of 1.5 times with that of 2 times, it was seen that the request blocking probability is slightly reduced at 1.5 times, but the difference is negligible. Overall, $D_{f} N_{m} 1.5$ showed the optimal performance.

\section{Conclusion}

Herein, we improved the evaluation formula of the Min-RDDR method and proposed a resource allocation method for AR requests in an EON. The method introduces a cost function that considers the condition of the adjacent link. We evaluated the method using the NSFNET topology. Results showed that the blocking probability was reduced by the proposed and normalized methods. Furthermore, we examined the coefficients and compared the blocking probabilities. Future studies involve shortening of the calculation time and studying the coefficients in terms of their interaction in the evaluation formula. 